\title{
Measurement of serum prostate cancer markers using a nanopore thin film based optofluidic chip
}

\author{
Salah Alzghoul ${ }^{1, *}$, Mohammad Hailat ${ }^{2, *}$, Sandra Zivanovic ${ }^{1}$, Long Que ${ }^{3, * *}$, Girish V. Shah ${ }^{2, * *}$ \\ Louisiana Tech University ${ }^{1}$, University of Louisiana-Monroe ${ }^{2}$, Iowa State University ${ }^{3}$ \\ *equal contribution \\ **Correspondence to G. Shah and L. Que (Email: lque@iastate.edu, shah@ulm.edu)
}




\begin{abstract}
Currently used cancer marker for prostate adenocarcinoma (PC), serum prostate-specific antigen (PSA), greatly overestimates PC population. Patients with high PSA levels have to undergo unnecessary but physically painful and expensive procedure such as prostate biopsies repeatedly. The reliability of PC test can be greatly increased by finding a protein that is secreted selectively by malignant, but not normal, prostate cells. A recently discovered novel protein, referred as neuroendocrine marker (NEM), is secreted only by malignant prostate cells and released in blood circulation. Although NEM seems to be significantly more reliable based on the data obtained from a limited cohort, currently available NEM ELISA is not suitable for undertaking a large study. Therefore, the goal of the present study was to develop an alternative, label-free assay system that can reliably measure NEM and PSA in patient samples. Herein an optofluidic chip that can reliably detect PSA as well as NEM in patient samples has been developed. The optofluidic chip, which consists of arrayed nanopore-based sensors fabricated from anodic aluminum oxide (AAO) thin film, offers improved sensitivity upon the optimization of the concentration of the detector antibodies immobilized on the sensor surface. The results demonstrate that the chip is reliable, extremely sensitive and requires just $1 \mu \mathrm{l}$ of patient serum (or even less) to measure PSA and NEM even in a non-cancer individual. Compared with the traditional ELISA for PSA, the nanopore-based sensor assay is 50-100 fold more sensitive, and offers many advantages such as elimination of labeled antigen, need for sophisticated equipment and highly trained individuals. These advantages, along with the low cost, should make the technology suitable for point-of-care application to screen elderly male populations for PC and to monitor the progress of patients undergoing PC treatment.
\end{abstract}

Key words: Nanopore-based sensor, optofluidic chip, serum-based biomarkers, prostate cancer 


\section{Introduction}

Prostate cancer (PC) is the second leading cause of cancer-related deaths in American men (Shui et al., 2014). Currently, prostate-specific antigen (PSA) is the only established biomarker for PC (Francini et al., 2014). However, PSA is secreted by normal as well as malignant prostates, and its levels reflect the size of the prostate rather than the cancer. Serum PSA levels increase in PC as well as benign diseases such as benign prostatic hyperplasia (BPH) and chronic prostatitis (Bangma et al., 2013; Cuzick et al., 2014; Kitagawa et al., 2014; Schroder and Roobol, 2009). As a result, the test based on the blood PSA levels grossly overestimates the number of PC patients, and all of them need to undergo additional test of prostate biopsy histology. It is estimated that almost a million men undergo prostate needle biopsy each year, but the cancer is detected in only a fifth of these men, of whom only a tenth may die of cancer (Bokhorst et al., 2013; Vickers et al., 2010). The availability of new cancer-specific markers can significantly reduce the large number of unnecessary prostate biopsies (up to 75\%), and help in better diagnosis and management of PC. We have identified a novel gene transcript, referred as Neuroendocrine Marker (NEM). Interestingly, NEM was detected only in malignant region, but not benign region, of the prostates; and NEM immunoreactive protein of $38 \mathrm{KDa}$ was also detected in human serum (Shah, 2011). Subsequently, we generated monoclonal and polyclonal antisera against NEM and established a nonequilibrium ELISA. Our preliminary study of a cohort of 69 PC and non-cancer patients revealed that serum NEM levels of PC patients were approximately three-fold higher than those with benign urologic diseases (Shah et al., 2009). The results suggested that NEM was more reliable than PSA in correctly identifying PC patients.

In a subsequent larger prospective study of over 500 patients (followed from 5 years prior to cancer detection), we also observed higher serum NEM levels in potential PC Patients as compared to noncancer individuals. However, the level of significance between PC vs benign patients declined due to lower precision of the NEM ELISA and larger interassay variations (Liang et al., 2011). These results 
suggested that the NEM ELISA was suitable for a small number of samples in a single assay, but needed significant improvements for larger applications. To overcome the instability of labeled NEM and the long procedure of non-equilibrium NEM ELISA assay, we aimed to develop a novel assay technology that was label-free, simple, quick, cost-effective and reliable. We present here an extremely sensitive assay technology that overcomes the deficiency of NEM ELISA, and uses microfluidics technology to concurrently measure NEM and PSA levels. Our results suggest that the new technology is suitable for the analysis of large number of samples on point-of-care basis.

\section{Materials and Methods}

\subsection{Fabrication of the nanopore thin film-based optofluidic chip}

The fabrication process of the anodic aluminum oxide (AAO) nanopore thin film sensor has been described in detail (He at al., 2014; Li et al., 2014; Li et al, 2013; He et al., 2012). This chip, consisting of arrayed nanopore-based sensors, has been fabricated using a newly developed process (Yin et al., 2014, Che et al., 2015). The fabrication process allows fabricating arrayed nanopore-based sensors on glass substrate in a very cost-efficient manner, rendering the disposable nanopore-based sensing chip possible. Briefly, the nanopores are formed in Al thin film by a two-step anodization process using electrolytes such as oxalic acid. After the AAO thin film is formed, the arrayed AAO patterns are fabricated by combining optical lithography and wet etching process. Then a layer of Au thin film (50 ̊) was coated on the AAO surface with a layer of $\mathrm{Cr}(5 \AA)$ as an adhesion layer. A polydimethylsiloxane (PDMS) microfluidic chip was fabricated separately using a soft lithography process and then was bonded with the AAO-glass chip, followed by assembling input and output tubing. A photo of the optofluidic chip, which consists of arrayed nanopore-based sensors is given in Fig. 1a. The SEM image of the fabricated AAO is shown in Fig. 1b-c.

\subsection{Instrumentation}


The detection setup in Fig. 1S (supplementary document) of the nanopore-based sensors is the same as those previously reported (Zhang et al. 2010; Zhang et al., 2011). Specifically, a broadband light source from a tungsten halogen lamp is coupled to a specifically-designed optical fiber probe (Ocean Optics, Inc., Dunedin, FL, USA), which illuminates the sensor perpendicularly. The reflected signals (transducing signals) are collected by the same optical fiber probe, leading to an optical spectrometer (Ocean Optics, Inc.), which can detect the optical spectrum from $350 \mathrm{~nm}$ to $1050 \mathrm{~nm}$. The spectrometer is connected to a laptop computer for data acquisition and processing. The biochemical samples are transported to the chip through the assembled plastic tubing (Upchurch Scientific, Inc.) by a syringe controlled by a pump (KD Scientific, Inc., Holliston, MA, USA).

\subsection{Chemicals and materials}

11-Mecaptoundecanoic acid (HSC10COOH, 99\%), 8-Mercapto-1-Octanol (HSC8OH, 98\%), N-(3Dimethylaminopropyl)-N'-ethylcarbodiimide hydrochloride, N-Hydroxysuccinimide (NHS), and glycine were purchased from Sigma-Aldrich (Milwaukee, WI) and used without further purification. Mouse antihuman prostate-specific antigen (PSA) monoclonal antibody (detector mAb) (cat. \# ABIN969369, clone \# 5A11E9, Antibody-online Inc., Atlanta, GA), The synthetic PSA peptide containing the middle region of PSA (LRPGDDSSHDLMLLRLSEPAELTDAVKVMDLPTQEPALGTTCYASGWGSI) was purchased from AVIVA Systems Biology, San Diego, CA). The stock solution of $1 \mathrm{mg} / \mathrm{ml}$ was prepared and stored at $-20{ }^{\circ} \mathrm{C}$ in small aliquots. Each aliquot was used for every assay and was diluted to the required concentration with the assay buffer. Absolute ethanol was obtained from Thermo Fisher Scientific Inc., USA. Deionized (DI) water was obtained from a DI water purification system (Millipore, FRANCE). Bovine serum albumin (BSA) was obtained from Bio-Rad Laboratories (Hercules, CA). It was diluted with PBS ( $\mathrm{pH}=7.2)$ solution at a concentration of $1.5 \mathrm{mg} / \mathrm{ml}$ for the control experiments. NEM monoclonal antibody and standard NEM peptide were custom prepared by our laboratory. The solutions of NEM were similar to those described for PSA. Serum samples of urology patients were left over from 
an earlier study that investigated NEM as a potential PC biomarker (Shah et al., 2009). The samples were leftover after the PSA assay of patients attending urology clinic at the VA Medical Center, Gainesville, FL. The protocol for the use and analysis of the sera was approved by the Institutional Review Boards at both institutions.

\subsection{Biodetection procedures}

Surface functionalization (Fig. 2S in the supplementary document): First, the detector mAb was immobilized on the gold-coated surface of the nanosensor as described before (Zhang et al., 2012). In brief, the gold-coated AAO surface was first cleaned with $\mathrm{O}_{2}$ plasma for 15 minutes before being bonded with the PDMS microfluidic layer. This was followed by a self-assembled monolayer (SAM) process of incubating the sensor in a mixture of $1 \mathrm{mM} \mathrm{HSC} 10 \mathrm{COOH}$ and $\mathrm{HSC} 8 \mathrm{OH}$ with molar ratio of 1:10 in absolute ethanol solution overnight. The SAM was activated by incubation in phosphate buffer solution (PBS; $10 \mathrm{mM}, \mathrm{pH} 7.0$ ) containing $0.5 \mathrm{mM}$ of EDC/NHS for 2 hours. The activated SAM was rinsed with the $10 \mathrm{mM}$ PBS, and then incubated with a freshly prepared $10 \mathrm{mM}$ PBS solution containing $10 \mu \mathrm{g} / \mathrm{ml}$ of the detector $\mathrm{mAb}$ for 2 or 4 hours. This incubation time could be significantly reduced to tens of minutes after optimization. It has been found that the $10 \mu \mathrm{g} / \mathrm{ml}$ of detector mAb provided best sensitivity among other concentrations including $1,2,5,10,20,40,60 \mu \mathrm{g} / \mathrm{ml}$. Hence the $10 \mu \mathrm{g} / \mathrm{ml}$ detector $\mathrm{mAb}$ has been used for all the experiments. The device was then rinsed with the PBS, followed by $0.2 \mathrm{M}$ glycine PBS solution for 10 min to deactivate the remaining active sites at the SAM. This avoided non-specific binding between the SAM and the antigens.

\subsection{Experimental data acquisition}

The average shift of the fringes for the measured transducing signals is obtained by (i) first obtaining the shift of each fringe peak relative to that of the blank Au-coated AAO surface or the shift after the antibodies (mAbs) have been immobilized on the AAO surface, then (ii) averaging the shift of all the peaks. The reference for each average shift is specified in the context in the following sections. 


\subsection{Immunoassay development}

The assay for two PC markers, NEM and PSA, was developed. PSA is the currently accepted serum PC marker ((Berges and Oelke, 2011; Yang et al., 1992). Serum PSA of 4 to $10 \mathrm{ng} / \mathrm{mL}$ suggests the possibility of early stage prostate cancer (Catalona et al., 2000; Liang et al., 2011; Raaijmakers et al., 2004), while normal levels are typically 0.5 to $3 \mathrm{ng} / \mathrm{ml}$. NEM is a unique antigen that was selectively expressed in malignant, but not benign, prostate. Our earlier results suggest that NEM is over-expressed in PCs, and its levels in cancer patients elevate to several-folds higher than those found in sera of noncancer individuals (Liang et al., 2011; Shah et al., 2009). Prior to the introduction of a sample into a chip, the microfluidic chamber of the sensor (capacity of $50 \mu \mathrm{l}$ ) was filled with $1.5 \%$ BSA to block nonspecific binding on all surfaces. This was followed by a washing with $0.05 \%$ Tween $20-\mathrm{PBS}$. Analyte proteins (PSA or NEM or sera) were then introduced in the chamber and incubated for 45 min. Our preliminary studies suggest that this time period is optimal for capture of the antigens by the antibodiescoated sensors. After final sequential washes, the readings were taken as described. All assay parameters including concentrations of the markers and incubation times were optimized to obtain high sensitivity and high signal to noise ratio. Negative controls employed the full immunoassay procedure but without the antigens (PSA and NEM). $50 \mu 1$ of different concentrations of NEM (0-32 pg) prepared in Sample Preparation Buffer (PBS, pH $7.4+1 \%$ Bovine Serum Albumin) were added into the sensor. Similarly, each dilution of serum samples $(0.1-10 \mu \mathrm{l}$ in a total volume of $50 \mu \mathrm{l})$ was also added in individual sensors. Positive controls were the aliquots of pooled serum of prostate cancer patients. The sensors were incubated at a room temperature for 45 minutes, washed with PBS and the average shift of the optical fringes for each sensor was determined by a spectrometer as described in Section 2.5. Similarly, the standard curves for PSA were obtained.

NEM ELISA: ELISA microtiter plates were coated with $23 \mathrm{ng}$ NEM antibody per well overnight at $4{ }^{\circ} \mathrm{C}$. Next day, the plates were washed in TBS-Tween 20 and blocked with $2 \%$ BSA solution. After several 
washes, the samples were added in the wells and incubated for 24 hours. Next day, the biotinylated NEM ( $1 \mathrm{pg} /$ well) was added and the incubation was continued at $37{ }^{\circ} \mathrm{C}$ for 3 hours. After several washes, streptavidin-horse radish peroxidase (HRP) conjugate was added and the incubation continued for 1 hour at $37^{\circ} \mathrm{C}$. After three washes, the HRP substrate (SuperSignal ELISA Femto, Thermo Fisher, Rockford, IL) was added, and chemiluminiscence was read on a Plate Reader.

\subsection{Validation}

For the validation of the sensors, key assay parameters such as specificity, sensitivity, linearity, accuracy, precision (intra and inter assay) and robustness were critically evaluated as per the requirements of international guidelines (Rodriguez et al., 2010; WHO, 1997). First, whether the presence of high concentration of unrelated protein(s) influences the binding between the detector antibody and the sensor was tested. The binding of NEM mAb or PSA mAb with the sensor in the presence/absence of BSA $(0.15$ $\mathrm{mg} / \mathrm{mL}$ ) was compared. Second, multiple sensors were used to determine the standard curve for NEM or PSA, and the results of different batches of sensors were compared to determine the reproducibility of the sensors. Third, aliquots of a serum pool (prepared by mixing sera of normal individuals) were used in multiple assays to determine the intra- and inter-assay imprecision. All samples were tested in duplicate. Generally, the coefficient of variation (CV) among the replicates was less than $7 \%$. However, the upper limit for the acceptance of CV was set at $20 \%$.

Recovery of added Antigen: To determine the accuracy of the sensor measurements, known amounts of NEM or PSA antigens were added into pooled serum aliquots. The amount of the antigen(s) determined by sensors was compared with the expected amounts of antigen.

Comparison of nanosensors with ELISA: The serum samples, left over from an earlier study that used ELISA to detect the antigens, were used in sensors assay. The results from both studies were compared.

\subsection{Statistics}

Imprecision of the assays is indicated as percentage coefficients of variation (\%CV). Influence of pre- 
analytical factors was calculated by the percentage recovery with respect to standard sample treatment.

\section{Results}

\subsection{Sensor surface functionalization and characterization}

The sensor was subjected to step by step surface functionalization procedure of the Au-coated AAO surface as described before (Zhang et al., 2012). This well-established procedure allows the formation of a mixed SAM of alkanethiols by the adhesion reaction of the thiol group on Au-coated surface (Cass, 1998). After the addition of EDC/NHS on the surface, the carboxylic groups form active O-acylisourea intermediates, and readily react with primary amine groups that exist at the $\mathrm{N}$-terminus of each polypeptide chain and in the side chain of lysine (Lys, K) residues. Due to their positive charge at physiologic conditions, primary amines are usually outward-facing of proteins. Hence, they are usually accessible for conjugation without denaturing protein structure. As a result, the detector mAbs for NEM or PSA can be covalently attached to the top of the mixed SAMs. In order to eliminate or mitigate the non-specific biological binding, the remaining active $\mathrm{O}$-acylisourea intermediate groups are deactivated by the amino acid glycine. Thereafter, the mAbs are conjugated to the sensor surface and ready for detecting NEM or PSA.

Representative measurement of the shift of the optical fringes for detecting NEM in buffer solution and the measurement of the specificity/selectivity of the bioassay are given in Fig. 2. The typical incubation time between the NEM Ab-coated sensor with NEM was 45 minutes. We observed that this incubation time is sufficient for optimal NEM-NEM Ab binding. After the incubation, the sensor received rigorous PBS buffer rinsing to remove unbound or loosely bound NEM before the optical measurements. Typical transducing signals before and after NEM binding are given in Fig. 2a. Clear shift of the interference fringes before and after NEM binding is observed due to the binding of NEM to its Abs. It has been found consistently that the larger concentration of the NEM samples, the bigger shift of the fringes. Indeed, no 
additional shift will be observed when all the available binding sites have been occupied by NEM. We reached that saturation point at $128 \mathrm{pg}$ of NEM or PSA when we applied the NEM or PSA Abs at $10 \mu \mathrm{g} / \mathrm{ml}$. However, one could increase this detection range by increasing the number of binding sites, i.e. by increasing the amount of Abs on the sensor.

To test the specificity of this method, the shift of the fringes when NEM solution was applied to the sensor versus concentrated bovine serum albumin (BSA) solution $(15 \mathrm{mg} / \mathrm{mL})$ has been compared. After 1-hour incubation, the sensor was rinsed with PBS buffer. The measurements in Fig. 2b demonstrate no clear shift of the transducing signals before and after the BSA application. In contrast, NEM solution gave clearly observable shift (Fig. 2a), demonstrating that the sensor can specifically detect the antigen even in the presence of large protein concentrations as long as the antibody is specific.

\subsection{Immunoassay development}

Initial studies examined the wider range of antigen concentrations (typically 0-256 pg PSA/NEM in $50 \mu \mathrm{l}$ sample) to find the most sensitive range for the sensors, which was in the range of 0 to $32 \mathrm{pg}$ in the calibration curves. Subsequently, 0-32 pg range for both markers was routinely used. The response of each data point was measured in triplicate to obtain the average wavelength shift. Typically, the average wavelength shift increased exponentially up to six-fold from the baseline in our standard curve range (Fig. 3a-b). When average wavelength shift was plotted against log (antigen concentrations), the curve showed sigmoid shape (Fig. 3a-b). Detection limits (DL) were measured as 3 times the average standard deviation (SD) above the zero biomarker control. It has been found that the DLs for the markers were 1 pg for PSA and $0.1 \mathrm{pg}$ for NEM, respectively.

The NEM antibodies were checked for cross-reactivity with multiple neuroendocrine peptides detected in the prostate and did not cross-react significantly with thyroid-stimulating hormone, neuropeptide Y, peptide YY, gonadotropin-releasing hormone, chromogranin A, calcitonin, and calcitonin gene-related peptide. In addition, negligible cross reactivity of NEM towards PSA antibody and vice versa has been 
observed in the experiments.

To test the reproducibility of the sensors, the assays with normal standard curves were performed on three randomly selected sets of chips, and run under the same conditions on three different days. Figure 3c demonstrates the reproducibility of the response. Average standard deviations were $11 \%$ at $1 \mathrm{pg}, 12 \%$ at 2 $\mathrm{pg}, 5 \%$ at $4 \mathrm{pg}$ and $6 \%$ at $8 \mathrm{pg}$ for NEM.

\subsection{Dilution curve with pooled human serum: Parallelism with the calibration curve}

To investigate whether our assay can detect the concentrations of a biomarker reliably in a serum sample, the parallelism between the dilution curves of NEM standard and serum samples was examined. We ran the pooled serum at various dilutions up to $10 \mu \mathrm{l}$ along with the calibration curves of NEM or PSA. The concentration of each antigen was calculated. Table 1 shows that the calculated value of NEM almost doubled with doubling of serum volume at least up to $10 \mu$ l. Corresponding volumes for PSA were 0.1$0.4 \mu \mathrm{l}$ for each PSA concentrations. Considering that the sensor can typically detect endogenous NEM/PSA levels in a volume of $0.1 \mu 1 / 50 \mu \mathrm{l}$ total volume, the interference by serum components in this system will be minimal even if it occurs.

\subsection{Reproducibility/Accuracy of the sensors:}

The reproducibility and accuracy of sensor were tested in two ways. First, the intraassay and interassay variations of same samples in an individual assay or in serial assays were evaluated. Secondly, pools of sera obtained from normal subjects were prepared, and the known amounts of antigen (either NEM or PSA) were added. Then, the samples were analyzed for the antigen (NEM or PSA) in a single assay. The detected value of added antigen was determined. The recovery varied from $92 \%$ at the lowest added concentrations ( 1 pg added PSA) to $69 \%$ at the highest added concentration (4 pg added PSA). Corresponding recovery of similarly added NEM was in the range of $75-79 \%$. Duplicates of these sample pools in subsequent assays were run to determine intra- and inter-assay variations. Repeated estimation of pooled serum in a single assay revealed imprecision of $7.4 \%$ for PSA and $6.8 \%$ for NEM in within-assay 
comparisons; and the same over three assays was $17.3 \%$ for PSA and $14.29 \%$ for NEM ( $\mathrm{n}=3)$ in betweenassay comparisons.

\subsection{Comparison of NEM sensor assay with NEM ELISA}

The NEM ELISA standard curve (Figure 4a) was compared with the NEM immunosensor standard curve

(Figure 4b). The results suggest that the immunosensor was approximately 50-100 fold more sensitive than the corresponding non-equilibrium ELISA. This, when considered together with the simplicity of the method, label-free assay and very short incubation period of 45 minutes, immunosensor offers significant advantages over the ELISAs for biomarker measurements.

\subsection{Assay validation with human serum samples}

10 serum samples from patients visiting the urology clinic for either BPH or PC leftover from the earlier study were analyzed (Shah et al., 2009). The serum NEM results obtained by the sensors were then compared with those obtained by NEM ELISA (Fig. 4c-d). There was a high correlation between serum NEM/PSA values determined by the sensor and those by the ELISA. More importantly, all PC samples with $>4 \mathrm{ng} / \mathrm{ml}$ PSA $(\mathrm{PSA}<4 \mathrm{ng} / \mathrm{ml}$ serum are considered non-cancer whereas PSA $>4 \mathrm{ng} / \mathrm{ml}$ serum are considered as potentially cancer) were also predicted to be the same by the current immunosensor assay. Then, the NEM and PSA levels measured by the sensors in these samples were compared with clinicopathological status of the patients as shown in Fig. 5. As expected, confirmed PC patients (Sample numbers $4,6,9)$ displayed high PSA (> 4ng/ml) as well as NEM (> $4 \mathrm{ng} / \mathrm{ml})$ levels. Among the remaining patients, we observed some divergences between NEM and PSA levels. For example, patients 7 and 10 displayed 4.59 and $4.39 \mathrm{ng}$ PSA/ml respectively. Based on the current cut-off, these patients are considered as potential cancer patients. However, their NEM levels were very low-1.762 and $2.096 \mathrm{ng} / \mathrm{ml}$ respectively. In contrast, patient \#1 and 2 displayed normal PSA levels (2.46 and $2.20 \mathrm{ng} / \mathrm{ml}$ respectively). However, their NEM levels were very high (6.523 and $13.187 \mathrm{ng} / \mathrm{ml}$ respectively). Although these results are very preliminary and much larger number of patients needs to be evaluated, the 
results raise a possibility that NEM may detect PC earlier than PSA (perhaps in case of \#1,2) or may detect indolent PC (in case of \# 7, 10).

\section{Discussion and Conclusion}

Present results demonstrate that the novel immuno-sensor can reliably detect prostate cancer biomarkers in ultralow volume of serum samples. The results also demonstrate that the sensor offers several advantages over the traditional ELISAs. First, the sensor has very low detection limits and the assay range for both the markers was 50-100-fold lower than that achieved by traditional ELISAs. Second, the sensor measures antibody-bound protein by interference optical signals coupled with localized surface Plasmon resonance (Zhang et al., 2010). This method completely eliminates the need for an enzyme-labeled antigen, or the requirement for efficient competition between labeled antigen and native molecules. There are serious difficulties in labeling NEM peptide with an enzyme conjugate that is thousand times larger than the NEM peptide. The labeled NEM is highly unstable, denatures rapidly and has difficulty competing with the native protein for antibody binding sites, leading to unacceptably high non-specific binding. Finally, the sensor technology also offers several other advantages such as simplicity of the procedure, significant reduction in the number of processing steps, and completion of the whole assay in a short time.

For any new detection technique, it is critical to demonstrate the technology measures the native molecules accurately, specifically and precisely in a complex mixture of potentially interfering proteins normally found in biological fluids. The accuracy of the sensor has been tested in multiple ways. First, whether high protein concentrations found in biological fluids such as serum would interfere with the measurement of biomarkers has been tested by comparing the calibration curve of biomarkers in normal buffer with that containing $0.15 \%$ bovine serum albumin. Identical curves have been found under both conditions, suggesting that high levels of albumin did not interfere with biomarker measurements. 
Second, whether the dilution curve of the patient serum is parallel to the calibration curve of the biomarker has been examined. Again, the dilution curve of the serum (up to $4 \mu \mathrm{l}$ ) parallel to the calibration curves of both biomarkers was observed. Third, the known concentrations of biomarkers were added in a serum sample, and whether the sensor can measure the added biomarker accurately has been tested. Again, the results show 70-107 \% of the added antigens was recovered in a serum sample at multiple concentrations. Fourth, PSA levels of ten patient samples of PC and benign urological diseases were measured by the sensor as well as the ELISA. The results of both methods are very similar (spearman's constant $\mathrm{r}=0.951$ ). Fifth, the serum PSA levels of the patients with their clinical diagnosis based on physical examination and pathology have been compared. As expected, confirmed PC patients displayed high levels of both markers. Based on these observations, it appears that the sensor is reliable, accurate and precise in typical analytical conditions for clinical measurements.

The correlation between NEM with PSA in same samples has been examined. In eight out of ten samples, very high correlation between PSA and NEM has been observed. Remarkable divergences between NEM and PSA in some samples have also been observed. Since the number of cases examined is low and we do not have access to the current status of the patients, we cannot say whether NEM can detect PCs with low PSA levels. However, there are two major gaps in the detection of PC. First, the most aggressive cases of PC are neuroendocrine type and do not display high levels of PSA (Lee et al., 2010; Trotz, 2003). Therefore, such cases are undetected by PSA test. On the other hand, PSA test significantly overestimates the number of cases with PC, resulting in false positive cancer diagnosis and associated undesirable consequences (Bokhorst et al., 2013; Vickers et al., 2010). Based on earlier studies and very limited data of the present study, it is likely that the NEM test may improve PC detection in both important areas. Indeed, the present study is designed to test the validity of an immuno-sensor for the measurement of PC biomarkers. However, these preliminary results demonstrate that the issues we faced with the NEM ELISA can be resolved with the current method, and should enable us to undertake a major study to 
investigate whether the dual NEM/PSA test can address the current deficiency in the field of prostate cancer detection. Indeed, this study will have larger patient cohort and well-defined statistical objectives. Although these studies are preliminary, it has been demonstrated that this nanopore-based sensor, fabricated by a low-cost standard micro- and nano-fabrication process (Yin et al., 2014), can be a viable prototype for future prostate cancer diagnostics in the clinic. After full development, the nanopore-sensor enabled system can be essentially accessible to virtually any biomedical laboratory at a low cost.

In summary, a novel, low-cost nanopore-based sensor has been developed for the detection of two biomarker proteins for prostate cancer. To our knowledge, this is the first demonstration of this type of sensor capable of detecting dual PC biomarkers with ultrasensitivity. The study presents the preliminary proof of principle for the reliable platform for developing clinically useful protein detection devices that could in future be translated to point-of-care in prostate cancer diagnostics and therapeutics.

\section{Acknowledgements}

The research was funded in part by NSF grant ECCS 0845370 and NSF-Pfund 2013 (to LQ), and by NIH grant R01CA096534 and Mary E. and Darrel Calhoun Endowment (to GVS). 


\section{References:}

Bangma, C. H., Bul, M., van der Kwast, T. H., Pickles, T., Korfage, I. J., Hoeks, C. M., Valdagni, R. (2013). Critical reviews in oncology/hematology, 85(3), 295-302.

Berges, R., Oelke, M., 2011. World journal of urology, 29(2), 171-178.

Bokhorst, L. P., Bangma, C. H., van Leenders, G. J., Lous, J. J., Moss, S. M., Schröder, F. H., Roobol, M. J., 2014. European urology, 65(2), 329-336.

Cass, T., Ligler, F. 1998. Immobilized biomolecules in analysis: A practical Approach. Oxford University Press, UK.

Catalona, W. J., Southwick, P. C., Slawin, K. M., Partin, A. W., Brawer, M. K., Flanigan, R. C., Bray, K. R., 2000. Urology, 56(2), 255-260

Che, X., He, Y., Yin, H., Que, L., 2015. Biosensors and Bioelectronics, 72, 255-260

Cuzick, J., Thorat, M. A., Andriole, G., Brawley, O. W., Brown, P. H., Culig, Z., Wolk, A., 2014. The Lancet Oncology, 15(11), e484-e492.

Francini, E., Petrioli, R., Rossi, G., Laera, L., Roviello, G., 2014. Tumour biology: the journal of the International Society for Oncodevelopmental Biology and Medicine. 35, 10601-10607.

He, Y., Li, X., and Que, L., 2014. Journal of biomedical nanotechnology, 10(5), 767-774.

He, Y., Li, X., and Que, L., 2012. Journal of nanoscience and nanotechnology, 12(10), 7915-7921.

Kitagawa, Y., Ueno, S., Izumi, K., Kadono, Y., Konaka, H., Mizokami, A., Namiki, M., 2014. Journal of cancer research and clinical oncology, 140, 53-59.

Lee, D.K., Park, J., Kim, J., Lee, S., Jo, M., Gil, M., Song, K., Park. J., 2010. Korean journal of urology, $51,358-361$.

Li, X., Yin, H., and Que, L., 2014. Biomedical Microdevices, 16(5), 771-777.

Li, X., He, Y., and Que, L., 2013. Langmuir, 29(7), 2439-2445.

Liang, Y., Ankerst, D. P., Ketchum, N. S.,Ercole, B., Shah, G., Shaughnessy, J. D., Leach, R. J., Thompson, I. M., 2011. J Urol. 185:104-110.

Raaijmakers, R., Blijenberg, B. G., Finlay, J. A., Rittenhouse, H. G., Wildhagen, M. F., Roobol, M. J., and Schroder, F. H., 2004. J Urol. 171, 2245-2249.

Rodriguez, H., Tezak, Z., Mesri, M., Carr, S., Liebler, D., Fisher, S., Tempst, P., Hiltke, T., Kessler, L., Kinsinger, C., Philip, R., Ransohoff, D., Skates, S., Regnier, F., Anderson, N., Mansfield, Workshop, P., 2010. Clin Chem. 56, 237-243. 
Schroder, F.H., Roobol. M. J., 2009. Current opinion in urology. 19, 227-231.

Shah, G. 2011. A human cancer cell specific gene product. USPTO, editor. Girish V. Shah.

Shah, G., Srivastava, A., Iczkowski, K., 2009. In 100th AACR Annual Meeting, Denver, CO.

Shui, I.M., Lindstrom, S., Kibel, A., Berndt, S., Campa, D., Gerke, T., Penney, K., Albanes, D., Berg, C., Bueno-de-Mesquita, H., Chanock, S., Crawford, E., Diver, W., Gapstur, S., Gaziano, J., Giles, G., Henderson, B., Hoover, R., Johansson, M.,Le Marchand, L., Ma, J., Navarro, C., Overvad, K., Schumacher, F., Severi, G., Siddiq, A., Stampfer, M., Stevens, V., Travis, R., Trichopoulos, D., Vineis, P.,Mucci, L., Yeager, M., Giovannucci, E., Kraft, P., 2014. Eur Urol. 65, 1069-1075.

Trotz, C., 2003. The Journal of the American Board of Family Practice / American Board of Family Practice. 16, 343-344.

Vickers, A.J., A.M. Cronin, G. Aus, C.G. Pihl, C. Becker, K. Pettersson, P.T. Scardino, J. Hugosson, and H. Lilja. 2010. Cancer. 116, 2612-2620.

WHO. 1997. Validation of analytical assays. In A WHO guide to GMP requirements. Vol. Part 2:

Validation. WHO, Geneva.

Yang, Y., Chisholm, G., Habib, F., 1992. Prostate. 21, 201-208.

Yin, H., Mu, A., Sun, Y., Yan, G., Wei, H., Ma, C., Sun C., 2014. Artificial cells, nanomedicine, and biotechnology, 1-7.

Yin, H., Li, X., and Que, L., 2014. Microelectronic Engineering, (128), 66-70.

Zhang, T., He, Y., Wei, J., Que, L., 2012. Biosensors and Bioelectronics. 38, 382-388.

Zhang, T., Pathak, P., Karandikar, S., Giorno, R., Que, L., 2011. Biosensors and Bioelectronics, 30(1), 128-132.

Zhang, T., Gong, Z., Giorno, R., Que, L., 2010. Optics express, 18(19), 20282-20288. 


\section{Figure caption:}

Fig. 1 (a) a photo of an optofluidic chip, consisting 15 AAO nanopore-based sensors; (b-c) SEM images of AAO inside each sensor.

Fig. 2 (a) Typical transducing signals (average shift of interference fringes $\sim 6.72 \mathrm{~nm}$ ) from the optical sensor after the NEM $(1.28 \mathrm{ng} / \mathrm{mL})$ is applied, incubated, and then rinsed using the buffer solution PBS in the sensor; (b) Specificity and selectivity test: transducing signal (negligible shift) from the sensor after BSA solution is flowed, incubated, and then rinsed using buffer solution PBS in the sensor.

Fig. 3 Immuno sensor assay characteristics: (a) a typical standard curve for NEM; (b) a typical standard curve for PSA; (c) NEM standard curves using three separate sets of sensors on separate days.

Fig. 4. Comparison between PSA immunosensor assay and ELISA: (a) a typical standard curve of NEM in an ELISA assay; (b) a typical NEM standard curve in an immunosensor assay; (c) linear regression curve between sensor vs ELISA for NEM (Pearson Co-efficient: 0.9507; P<0.0001); (d) linear regression curve between sensor vs ELISA for PSA (Pearson Co-efficient: 0.9431; P<0.0001).

Fig. 5. Analysis of NEM and PSA in serum samples of urology patients: Correlation between NEM and PSA in same samples (Pearson Co-efficient $\mathrm{r}=0.8557$; $\mathrm{p}<0.0016$ ).

Table 1: Calculated amounts of NEM and PSA used for experiments for obtaining parallelism between serum dilution curve and standard curve for NEM and PSA 

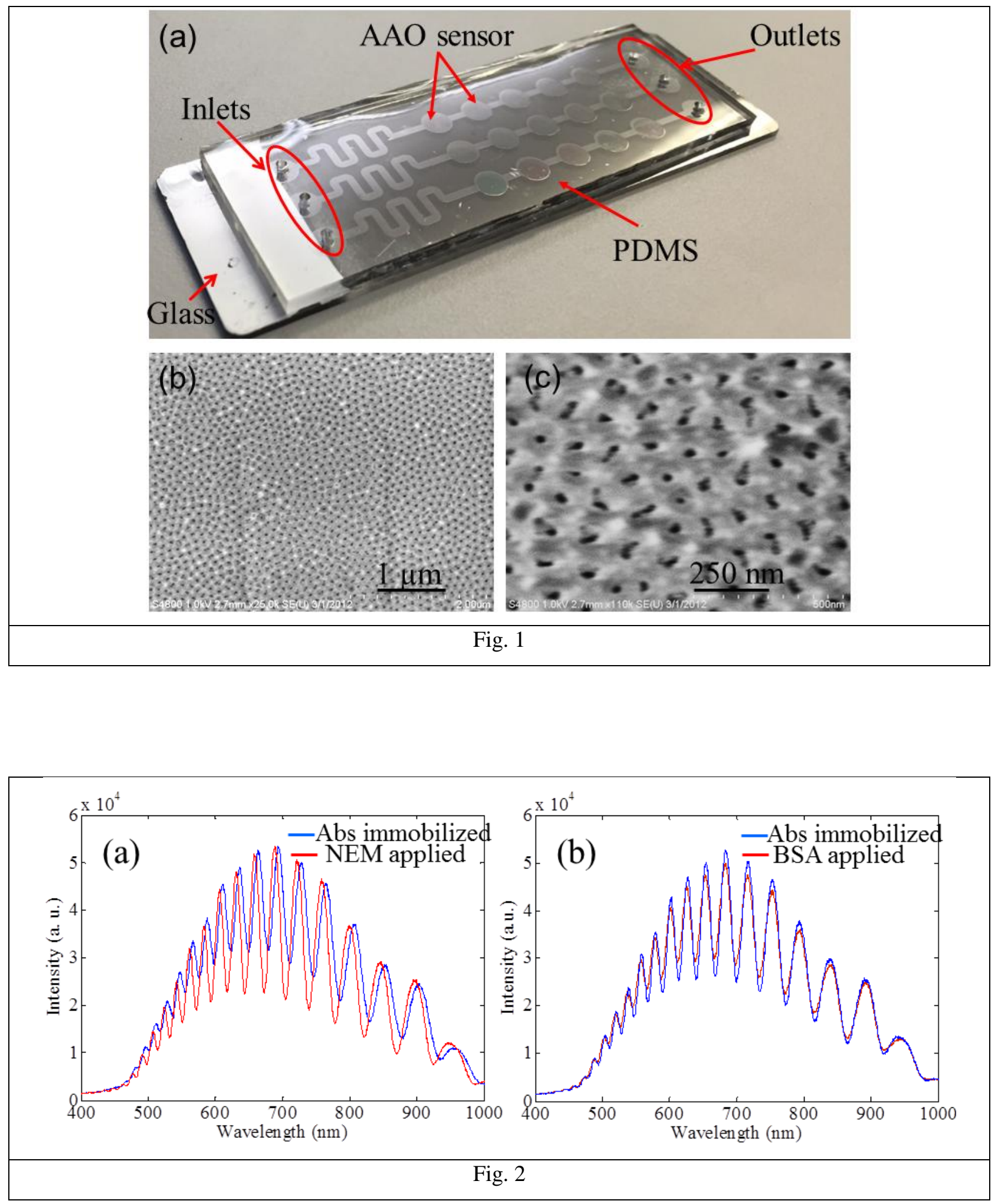


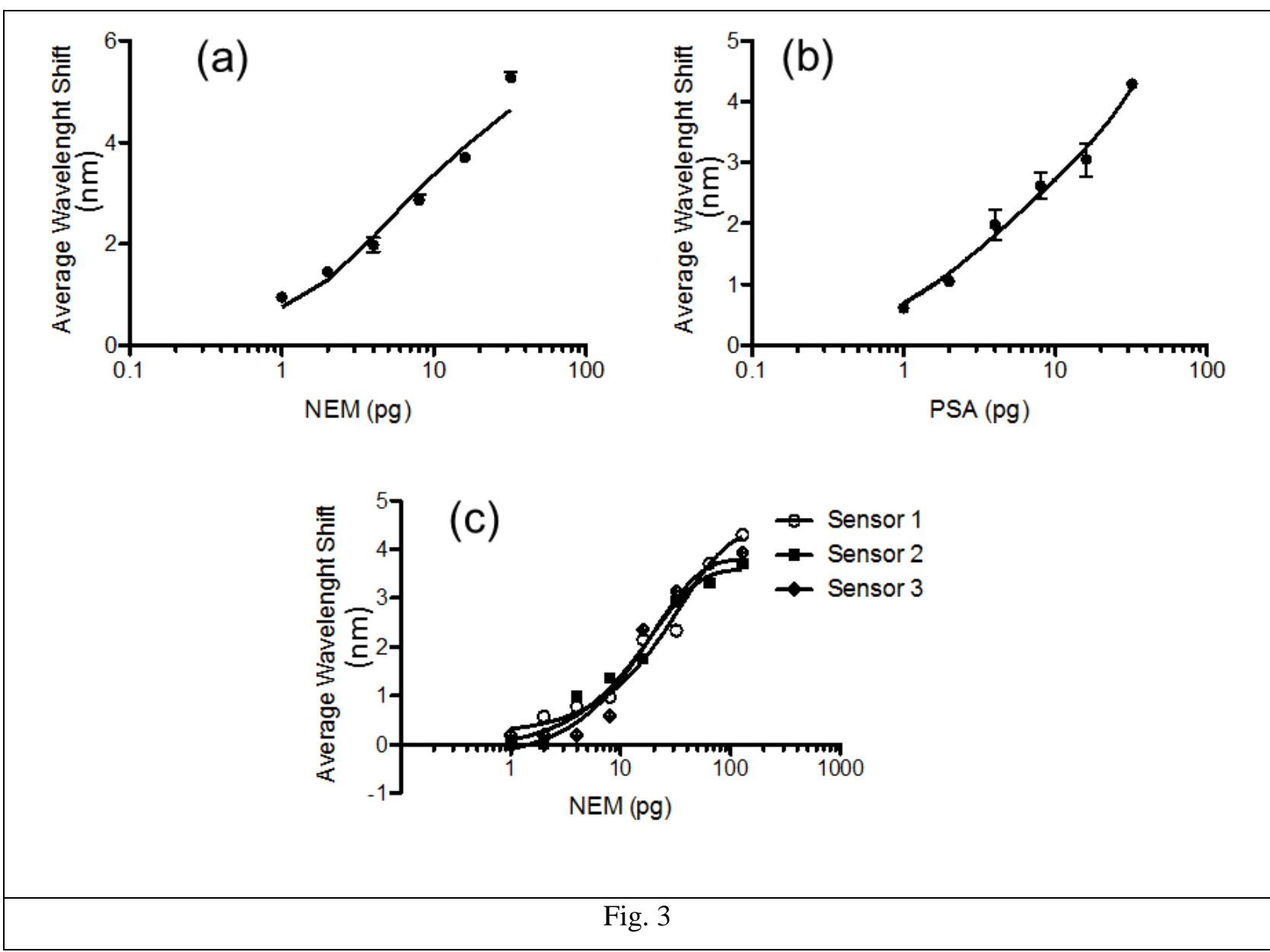



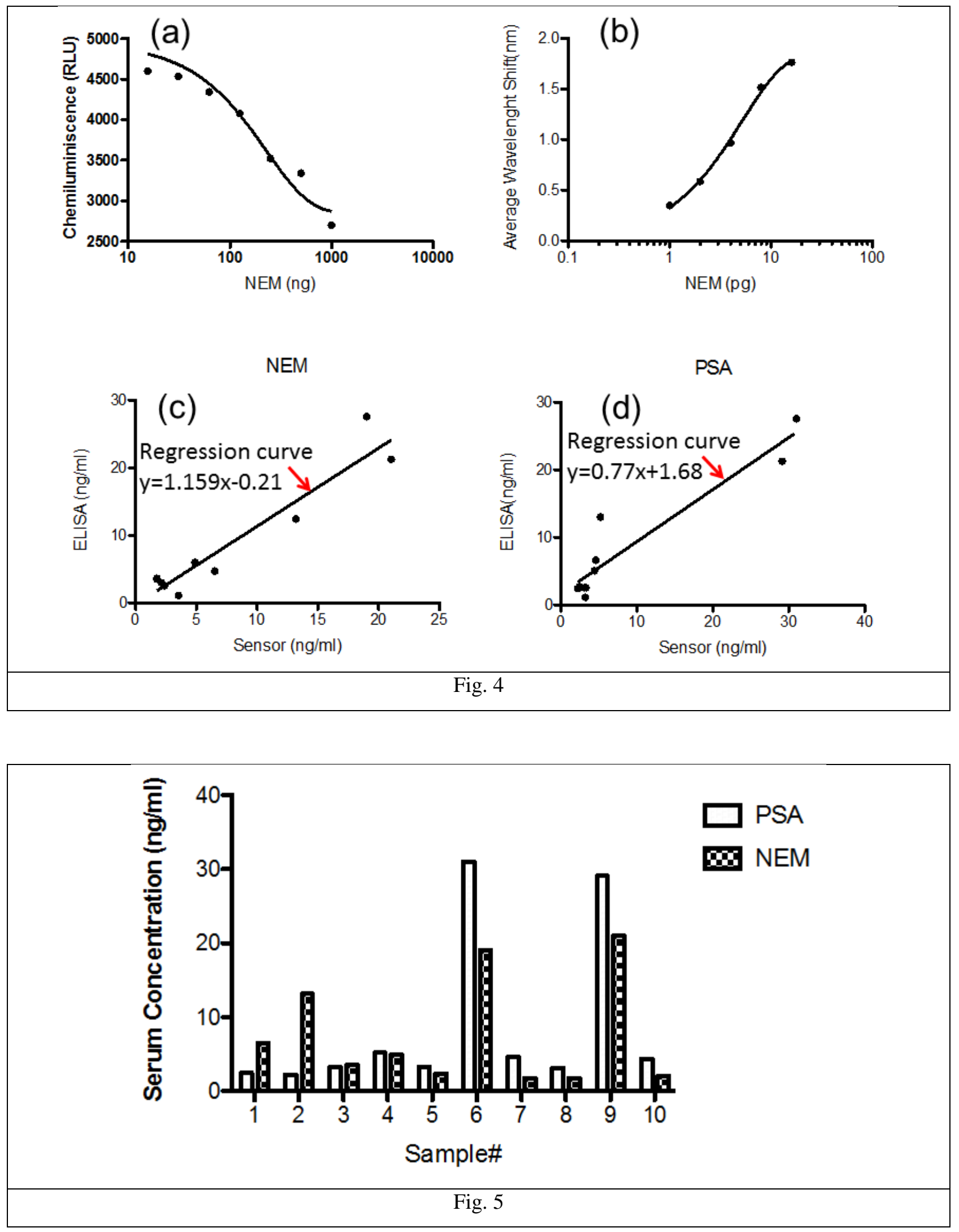

21 
Table 1

\begin{tabular}{|l|l|l|l|l|l|l|}
\hline $\begin{array}{l}\text { Serum sample } \\
(\mu \mathrm{l})\end{array}$ & $\begin{array}{l}\text { Calculated } \\
\text { NEM }(\mathrm{pg})\end{array}$ & $\begin{array}{l}\text { Increment: } \\
\text { NEM }(\mathrm{pg})\end{array}$ & & $\begin{array}{l}\text { Serum sample } \\
(\mu \mathrm{l})\end{array}$ & $\begin{array}{l}\text { Calculated } \\
\text { PSA }(\mathrm{pg})\end{array}$ & $\begin{array}{l}\text { Increment: } \\
\text { PSA }(\mathrm{pg})\end{array}$ \\
\cline { 1 - 2 } 1.25 & 9.5 & & 0.1 & 2.4 & \\
\hline 2.5 & 17.0 & 7.5 & 0.2 & 5.0 & 2.6 \\
\hline 5 & 31.6 & 14.6 & 0.4 & 9.3 & 4.3 \\
\hline 10 & 62.3 & 30.7 & & & \\
\cline { 5 - 6 } & & & & & \\
\hline
\end{tabular}

